\title{
Sphere Bubble Film of Poly(vinyl alcohol)
}

\author{
Kazuo YAmaura, Tetsuya TANigami, and Shuji Matsuzawa \\ Faculty of Textile Science and Technology, Shinshu University, \\ Tokida 3-15-1, Ueda, Nagano 386, Japan
}

(Received May 30, 1983)

\begin{abstract}
KEY WORDS Poly(vinyl alcohol)/Sphere Bubble Film / Stereoregularity / Adsorption / Colored Film / Thin Film /
\end{abstract}

In a previous paper, ${ }^{1}$ we showed that a very thin, sphere bubble film can be formed from a regenerated silk fibroin solution. Silk fibroin molecules ${ }^{2}$ and also poly(vinyl alcohol) (PVA) molecules ${ }^{3}$ are adsorbed easily at the air/water interface. For PVA $_{\mathbf{V A c}}$ derived from vinyl acetate, Imai and Matsumoto ${ }^{4}$ carried out a foam fractionation in which PVA molecules were adsorbed on fine foams produced by shaking an aqueous solution, and found that the fractionation was influenced by the stereoregularity of the PVA. Thus, there seemed to be the possibility of obtaining a sphere bubble film from aqueous solutions of PVA and silk fibroin. We attempted an experiment to confirm this possibility and also to examine the effect of the stereoregularity of PVA in the formation of a sphere bubble film.

A commercial PVA $_{V A c}$ derived from vinyl acetate

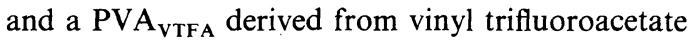
were used as the samples. The degree of polymerization $(D P)$ and the content of the syndiotactic diad of the $\mathrm{PVA}_{\mathrm{VAc}}$ sample were 1700 and $45 \%$, respectively, and those of the PVA $\mathrm{VTTA}_{\mathrm{VTF}}$ sample were 1570 and $55 \%$. A sphere bubble film was prepared as follows: An aqueous PVA solution of a fixed volume was sucked up into the tip of a glass tube with an inner diameter of about $1 \mathrm{~mm}$ and which was then blown by pushing out air in time intervals of $5-10 \mathrm{~s}$ by a pipetter. In the case of PVA $\mathrm{VAc}_{\mathrm{Ac}}$, liquid drops fell from the glass tube and no sphere bubble film could be prepared in a concentration range from 10 to $100 \mathrm{~g} \mathrm{dm}^{-3}$. However, a sphere bubble film was made successfully when aqueous PVA $_{\text {VTFA }}$ solutions in a concentration range from 10 to $35 \mathrm{~g} \mathrm{dm}^{-3}$ were pushed out slowly. Hence, the stereoregularity of PVA molecules plays an impor- tant role in the formation of a sphere bubble film. The sphere bubble film obtained from the PVA aqueous solution at concentration $c$ of $30 \mathrm{~g} \mathrm{dm}^{-3}$ was the most stable.

During the initial blowing, a sphere bubble film containing water was transparent, but during subsequent blowing, it began to change color at a certain diameter or thickness. Partial coloring of the film containing the solvent occurred during the initial blowing. When the entire sphere bubble film containing the solvent showed a color change during the blowing, the film became very weak and ruptured on further blowing. Table I shows the relation between the weight of a liquid drop and the diameter of a colored sphere bubble for the PVA $_{\text {VTFA }}$ aqueous solution of $c=30 \mathrm{~g} \mathrm{dm}^{-3}$ The thickness of a colored sphere bubble film containing water increased with decreasing volume of the solution. It seems that the color is related to the light-path length of the refracted rays in the film and the angle of incidence onto the film. A rainbow color was often found on the sphere bubble during the vaporization of water following blowing. The development of this color pattern seems related to the thining of the sphere bubble film following the vaporization of water. Vaporization of water from the film was complete after $5-10 \mathrm{~min}$ at a relative humidity of about $60 \%$ at room temperature (about $20^{\circ} \mathrm{C}$ ). A film apparently containing no water is referred to as a dried film in this note. Table I shows that dried films are very thin. The color of the sphere bubble film became light black at an extremely thin part of the film. Figure 1 shows a photograph of the sphere bubble free from water obtained from an aqueous PVA VTFA $_{\text {solution of }}$ 
Table I. The relation between the weight of a liquid drop and the thickness of film with or without solvent for $30 \mathrm{~g} \mathrm{dm}^{-3}$ aqueous $\mathrm{PVA}_{\mathrm{VTFA}}$ solution

\begin{tabular}{|c|c|c|c|c|}
\hline \multirow{3}{*}{$\begin{array}{c}\begin{array}{c}\text { Weight of } \\
\text { a liquid drop }\end{array} \\
\mathrm{g}\end{array}$} & \multicolumn{2}{|c|}{$\begin{array}{l}\text { Colored sphere bubble } \\
\text { containing solvent }\end{array}$} & \multicolumn{2}{|c|}{ Dried film } \\
\hline & Diameter & Thickness $^{\mathrm{a}}$ & Weight & Thickness ${ }^{\mathrm{a}}$ \\
\hline & $\mathrm{cm}$ & $\mu \mathrm{m}$ & $\mathrm{mg}$ & $\AA$ \\
\hline 0.0121 & 2.5 & 6.17 & 0.420 & 1660 \\
\hline 0.0242 & 4.8 & 3.34 & 0.842 & 940 \\
\hline
\end{tabular}

a These values were evaluated from the volumes of solution or dried film and the diameter of the sphere bubble. The volume of dried film was evaluated from the weight of an amorphous film.

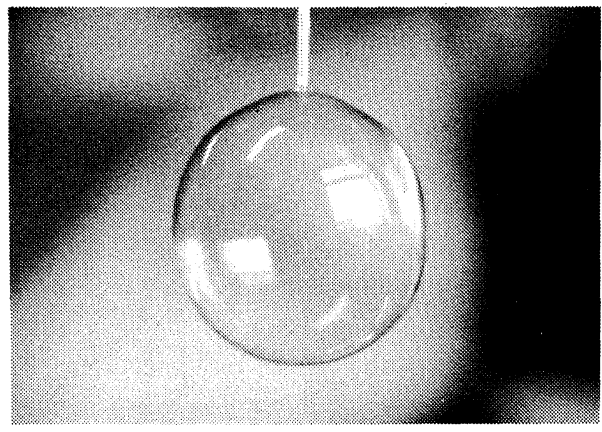

Figure 1. Photograph of a dried, sphere bubble obtained from aqueous PVA $\mathrm{VTFA}_{\text {A }}$ solution of $c=30 \mathrm{~g} \mathrm{dm}^{-3}$.

$c=30 \mathrm{~g} \mathrm{dm}^{-3}$.

At present, we have no definite concept of the conformation of the PVA $\mathrm{VTFA}_{\mathrm{VT}}$ molecules in the thin film, but should like to propose a structural model of the film on the basis of our previous results for the surface tension ${ }^{3}$ or the flow-induced crystallization $^{5}$ of aqueous solutions of PVA's with different tacticities. In this model, the hydrophobic parts (syndiotactic parts) appear on the film surface and the hydrophilic parts (isotactic and atactic parts) turn toward the film interior. If this is the actual situation, very thin films should have a bilayer structure similar to a soap membrane. The sphere bubble film showed no birefringence when observed under a polarizing microscope. The infrared spectrum of an untreated film showed only a small shoulder at $1140 \mathrm{~cm}^{-1}$ corresponding to the crystalline parts, but the spectrum of a film stretched to 3 times its original length had a stronger absorption peak at $1140 \mathrm{~cm}^{-1}$. The polarizing microscopic observation and the infrared spectra for very thin, sphere bubble films of $\mathrm{PVA}_{\mathrm{VTFA}}$ gave no evidence of a bilayer structure.

\section{REFERENCES}

1. K. Yamaura and S. Matsuzawa, Preprints of U.S.Japan Seminar on Polymer Liquid Crystals, Kyoto, 1983, p 259.

2. K. Yamaura, Y. Okumura, and S. Matsuzawa, $J$. Macromol. Sci., Phys., B21, 49 (1982).

3. S. Matsuzawa, K. Yamaura, N. Yoshimoto, I Horikawa, and M. Kuroiwa, Colloid Polym. Sci., 258, 131 (1980).

4. K. Imai and M. Matsumoto, Bull. Chem. Soc. Jpn., 36, 455 (1963).

5. Y. Go, S. Matsuzawa, Y. Kondoh, K. Nakamura, and T. Sakamoto, Kobunshi Kagaku, 25, 55 (1968); S. Matsuzawa, K. Yamaura, Y. Umeda, Y. Inoue, H. Kitamura, and S.-Y. Pak, Makromol. Chem., 180, 2009 (1979). 